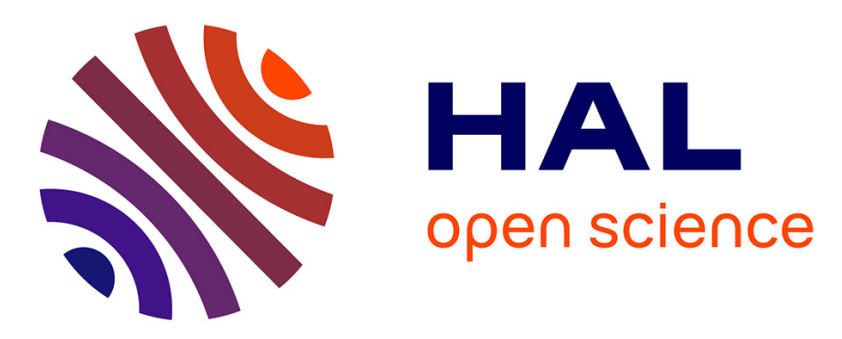

\title{
Towards a transparent deliberation protocol inspired from supply chain collaborative planning
}

Florence Dupin de Saint Cyr - Bannay, Romain Guillaume

\section{To cite this version:}

Florence Dupin de Saint Cyr - Bannay, Romain Guillaume. Towards a transparent deliberation protocol inspired from supply chain collaborative planning. International Conference on Information Processing and Management of Uncertainty in Knowledge-based Systems (IPMU), Jul 2014, Montpellier, France. pp. 335-344. hal-01118218

\author{
HAL Id: hal-01118218 \\ https://hal.science/hal-01118218
}

Submitted on 18 Feb 2015

HAL is a multi-disciplinary open access archive for the deposit and dissemination of scientific research documents, whether they are published or not. The documents may come from teaching and research institutions in France or abroad, or from public or private research centers.
L'archive ouverte pluridisciplinaire HAL, est destinée au dépôt et à la diffusion de documents scientifiques de niveau recherche, publiés ou non, émanant des établissements d'enseignement et de recherche français ou étrangers, des laboratoires publics ou privés. 


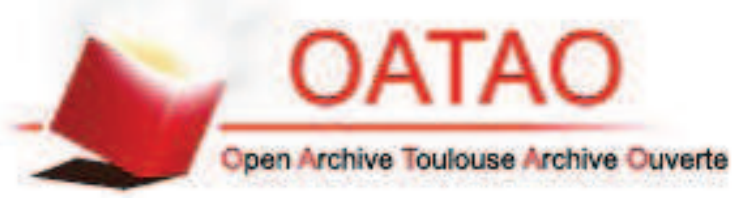

Open Archive TOULOUSE Archive Ouverte (OATAO)

OATAO is an open access repository that collects the work of Toulouse researchers and makes it freely available over the web where possible.

This is an author-deposited version published in: http://oatao.univ-toulouse.fr/ Eprints ID : 12984

To cite this version Dupin De Saint Cyr - Bannay, Florence and Guillaume, Romain Towards a transparent deliberation protocol inspired from supply chain collaborative planning. (2014) In: International Conference on Information Processing and Management of Uncertainty in Knowledge-based Systems (IPMU), 15 July 2014 - 19 July 2014 (Montpellier, France).

Any correspondance concerning this service should be sent to the repository administrator: staff-oatao@ listes-diff.inp-toulouse.fr 


\title{
Towards a transparent deliberation protocol Inspired from supply chain collaborative planning*
}

\author{
Florence Bannay $\quad$ Romain Guillaume \\ IRIT, Toulouse University, France
}

\begin{abstract}
In this paper we propose a new deliberation process based on argumentation and bipolar decision making in a context of agreed common knowledge and priorities together with private preferences. This work is inspired from the supply chain management domain and more precisely by the "Collaborative Planning, Forecasting and Replenishment" model which aims at selecting a procurement plan in collaborative supply chains.
\end{abstract}

Keywords: Decision process, Argumentation, Supply Chain Management.

\section{Introduction}

In the supply chain management (SCM) domain, collaborative planning is the process of finding a production plan which is suitable for every agent involved in the supply chain (SC). In this domain, a useful requirement is that the agents have agreed about some general conventions concerning the eligible criteria wrt production plans (avoiding more discussions about criteria). From Artificial Intelligence point of view, the SCM collaborative plan problem is a particular case of a collaborative decision given a background consensual knowledge and the particular preferences of each agent. This decision is the selection of a candidate according to a global convention and according to the precise arguments uttered by them about each candidate. In the classification of dialogs given by Walton and Krabbe [17], we are facing a deliberation process, since the initial situation is a dilemma (the agents need to find a good plan among several options), each agent aims at coordinating its goals and actions with the others in order to decide the best course of action that will be beneficial for all the participants.

In existing process, supply chain participants are compelled to provide numerical data (which may require some expensive computation, or may be based on some debatable numerical evaluations). Our main goal is to design a formal framework in which (qualitative) arguments in favor or against a plan maybe expressed and may interact with each-other. This framework will enable to conceive systems that could facilitate and guide the agents to converge rationally towards a set of consensual plans. Moreover, the production plan that will be chosen should have a guaranteed quality: if the selection leads to a set of plans that are under an admissibility threshold then the process is aborted and an exception is raised. Otherwise, if several plans are admissible then the customer (or the most important actor playing a role similar to a customer wrt a supplier ([13]) has usually the last choice. Thus, our aim is twofold, enlarge the expressive power and readability of the decision protocols concerning collaborative planning in SCM domains, import these ideas to AI field in order to propose a new rational model of collaborative decision making (or deliberation) under clear admissibility criteria.

More precisely we propose a new deliberation process based on bipolar argumentation where the arguments, their incompatibilities (called attacks) and their importance levels are commonly agreed before the deliberation. We directly apply this process for collaborative planning in supply chain using CPFR $\mathbb{R}$ ( Section 2.3). In AI literature some models have already been proposed based on a bipolar view of alternatives. Indeed, it is often the case that human people evaluate the possible alternatives considering positive 
and negative aspects separately [16]. Moreover, argumentation has already been proposed to govern decision making in a negotiation context (see for instance [2] and [14] for a survey). But, as far as we know, the use of bipolar argumentation in order to govern a deliberation process under instantiated arguments had never been studied. Moreover, in context of supply chain the agents only require to obtain admissible production plan and do not necessarily need to class them (in the worst case the best plan can be inadmissible, in other cases every plan may be admissible). Hence, we propose and study the rationality and the calculability of the admissible set. Furthermore, the notion of efficiency and simplicity that are central in SCM domain can bring a new perspective for modeling deliberation process in AI.

We first recall the basis of qualitative bipolar decision, classical argumentation and supply chain planning. In a second step, we define a common decision making structure gathering the concepts of bipolarity and argumentation, then we propose admissibility thresholds. We apply this deliberation process to an example of collaborative planning.

\section{Background}

\subsection{Qualitative bipolar decision}

In this paper we focus on the problem of qualitative bipolar decision, this problem can be formalized as follows. Let $C$ be a finite set of potential choices and $\mathcal{A}$ be a set of arguments (or criteria) viewed as decision attributes ranging on a bipolar scale $\{-,+\}$. More precisely if $a \in \mathcal{A}$ and $c \in C$ then $a(c)$ is either an argument in favor of the choice $c$ (when $a(c)=+$ ) or against it $(a(c)=-$ ). Let us consider a totally ordered scale level expressing the relative importance of arguments: $\forall a_{1}, a_{2} \in \mathcal{A}$, if level $\left(a_{1}\right)>\operatorname{level}\left(a_{2}\right)$ then $a_{2}$ is more important than $a_{1}$ (the best level is 1). In this paper, a set $A \subseteq \mathcal{A}$ of arguments is called a bipolar leveled set of arguments abbreviated bla, and $A^{+}=\{a \mid a(c)=+\}$ and $A^{-}=\{a \mid a(c)=-\}$ denote respectively the set of arguments in favor and against the choice $c$. The problem of rank-ordering the possible choices has been well studied in the literature. For this purpose, decision rules which build preference relations between decisions were proposed [4].

Most approaches in the literature focus on preferences but do not discus the problem of admissibility threshold. In this paper we propose such kind of thresholds.

Note that classical models do not take into account the conflicts that may occur between arguments, indeed the presence of some arguments may decrease the validity of other arguments. In this paper we propose a model that takes into account both the importance of arguments and the relations between them.

\subsection{Classical argumentation}

In the abstract argumentation theory, Dung [8] has defined several ways, called "semantics", to select admissible arguments. Given a graph $(X, R)$, called argumentation system where $X$ is a set of arguments and $R$ is a binary relation on $X$ called attack, the selected sets of arguments $S \in X$, called extensions, should be conflict free (i.e. should not contain internal attacks). Most of the extensions proposed by Dung are based on a defense notion ${ }^{1}$ that is not very intuitive in our context. Including defended arguments would amount to accept every positive argument as soon as one positive argument is not attacked (and similarly for negative arguments). Moreover, as already noticed by Amgoud and Vesic [2] "Dung's framework cannot be used for decision making since it simply selects groups of arguments containing at last, one of the strongest (positive or negative) arguments". Hence, Dung's framework is not useful in our context: it will give the same results with a more complex computation, as the results obtained by simply selecting the unattacked arguments.

\subsection{Supply chain planning}

A supply chain is "a network of connected and interdependent organizations mutually and cooperatively working together to control, manage and improve the flow of materials and information from suppliers to end users" [5]. Handling a supply chain is called supply chain management and can be defined as "the

\footnotetext{
${ }^{1}$ A set of arguments $Y$ defends an argument $x$ iff for any argument $y$ s.t. $(y, x) \in R$ there exists an argument $z \in Y$ such that $(z, y) \in R$.
} 
management of upstream and downstream relationships with suppliers and customers to deliver superior customer value at less cost to the supply chain as a whole" [5]. Two types of supply chains can be distinguished: decentralized supply chains (SC where actors are independents) and centralized supply chains (SC where decision authority and information is hold by one single party) [1]. In this paper we focus on decentralized supply chains. The key of successful SCM for decentralized supply chains is the collaborative process.

The collaborative processes are usually characterized by a set of point-to-point customer / supplier relationships with partial information sharing [7]: one or several procurement plans are built and propagated through the supply chain using negotiation processes. One of the most popular collaborative processes has been standardized under the name of "Collaborative Planning, Forecasting and Replenishment" $(\mathrm{CPFR} R)$ [10]. It is also a registered trademark of Voluntary Inter-industry Commerce Standards (VICS).

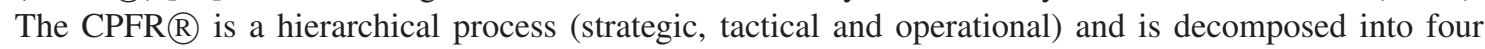
main tasks: Strategy and Planning, Demand and Supply Management, Execution and Analysis. In this paper, we are interested by the collaborative task of Demand and Supply Management, and more precisely by Order planning/forecasting and replenishment planning which are tasks that aim to determine future product ordering and some delivery requirements based upon the sales forecast, inventory positions, transit lead times, and other factors. In the literature of production planning, the collaborative order planning and replenishment planning are based on linear programming and/or mixed integer programming where the actors negotiate about a cost function which aggregates the inventory, the capacity, the production consequences ... (see [7] and [1] for a survey). Since some coefficients of the cost function are difficult to estimate for these actors, the analyses of resulting plans become difficult.

Another method, is to propose a set of plans to the supplier. Then the supplier evaluates the plans in terms of risk of back-ordering [9] and chooses the less risky plan. However, the risk of back-ordering is still poor information regarding the multi-dimension nature of evaluation of a production planning process [12].

Another part of SCM literature focuses on the actor planning process and on how to take into account the multi-objective nature of the problem and the qualitative evaluation of a production plan. For these purposes, the fuzzy goal programming approach [11] and the fuzzy multi-objective approach [3] have been proposed. The fuzzy goal programming approach consists in defining goals in terms of fuzzy intervals, the more the result belongs to the goal the more pleased is the decision maker. The fuzzy multi-objective approach takes into account the fuzziness of the importance of the criteria. For the problem of production distribution planning, a collaborative fuzzy programming approach has been developed in [15] which proposes a hierarchy of priority levels for the objective functions of the model, taking into account the dominance of each firm.

Those models do not take into account the bipolarity of decision. Our proposal aims to generalize the approach of [9] by taking into account the multi-objectives and qualitative nature of the problem using a bipolar argumentative framework.

\section{A Formal Framework for Bipolar Argumentative Deliberation}

A collaborative deliberation process involves several agents that want to select a candidate (or an option) in a "rational" manner. In order to do this selection, the agents are going to present arguments in favor or against some candidates. Then the system will aggregate their arguments and return the winner(s). The idea, borrowed from collaborative planning, is that the agents should first agree on general criteria in terms of arguments that may be used in favor or against a decision, and in terms of dominance/defeat relations between those arguments. After this agreement stage which is done once and for all, they can proceed to the selection of the candidates by giving arguments in favor or against some of them according to their private knowledge and preferences.

\subsection{Bipolar Leveled Features Set}

Let $C=\left\{c_{1}, \ldots c_{n}\right\}$ be a set of candidates (choices) and $V=\left\{v_{1}, \ldots v_{n}\right\}$ a set of voters, we are going to consider the arguments in favor or against the different candidates from the point of view of each voter. Arguments are entities representing a particular feature that may characterize some candidates, and influence 
their selection.

Example 1 In supply chain planning the problem is to find a production plan which satisfies both customers and suppliers. Thus, the set of candidates is the set of possible production plans and the customers and the suppliers are the voters. In this example, we consider three plans $c_{1}, c_{2}$ and $c_{3}$ and two voters $v_{1}$ (the supplier) and $v_{2}$ (the customer).

Definition 1 (instantiated argument) An instantiated argument $a_{v}(c)$ is a predicate based on an argument $a$, given $a$ voter $v$ and a candidate $c$. Let $I$ be the set of all instantiated arguments ${ }^{2}$, namely, $I=\left\{a_{v}(c) \mid\right.$ $v \in V, c \in C, a \in \mathcal{A}\}$. Moreover for any $c \in C, I(c)=\left\{a_{v}(c) \mid a \in \mathcal{A}, v \in V\right\}$ denotes the set of instantiated arguments about $c$.

Given a set of arguments the universe is the structure that contains those arguments together with their interactions.

Definition 2 ((instantiated) universe) $A$ universe (respectively instantiated universe) $\left(A_{U}, R_{U}\right)$ is a graph whose vertices are arguments $A_{U} \subseteq \mathcal{A}$ (respectively instantiated arguments $A_{U} \subseteq I$ ) and arcs are conflict relations between arguments such that $R_{U} \subseteq A_{U} \times A_{U}$.

If $R$ is an attack relation then $(a, b) \in R$ means that the argument $a$ defeats the argument $b$. In our context $a$ is a possible feature, thus $(a, b) \in R$ means that if $a$ characterizes a candidate then the fact that $b$ also holds for this candidate has no interest nor influence on the final result ( $b$ is invalidated by $a$ ).

Note that a universe is a Dung argumentation system [8]. Now, let us consider that the protagonists have agreed about a consensual universe. This consensual agreement concerns the features that will be used in arguments, it concerns also the way they are divided into two sets (features "in favor of" and features "against" the candidates). Finally the agreement between the participants concerns also the importance accorded to each feature, it is translated by a level, the higher level corresponds to the features that play the smaller role in the decision.

Note that the different features in $A^{+}$are arguments in favor of a candidate hence they are, by nature, opposed to the arguments against this candidate i.e. features in $A^{-}$(and conversely). This is why a bla will be associated with a graph in which each argument in $A^{+}$is attacking any less important $\operatorname{argument}$ in $A^{-}$ (i.e. belonging to a greater level).

For arguments situated at the same level $\lambda$, the relation is not so systematic. Let us denote by $A_{\lambda}$ the set of arguments at level $\lambda$, we consider that a relation $R_{\lambda}$ should be defined in order to express the attacks between $A_{\lambda}^{+}$and $A_{\lambda}^{-}$. These attacks are not necessarily symmetric. We can be confronted to three relationships between a positive and a negative feature of the same level:

- they may have a completely independent impact on the accuracy to select a candidate having these features: no attack (e.g. in the supply chain management domain, the carbon impact and the stability of the production plan)

- they may have an opposite impact: hence when they appear together the attack is symmetric (e.g., a plan that may be considered both satisfactory and not satisfactory for personal reason)

- they may be related in a way that the presence of the first one outperforms the other (although they have the same level of importance) for instance a plan that may imply a high inventory level for an actor of the supply chain is bad even if this plan makes a quick flow for an other actor.

This is why the relation $R_{\lambda}$ should be given for each level $\lambda$.

Definition 3 (universe associated to a bla) Let $A$ be a bla with l levels. The universe associated to $A$ given a family of l binary relations on $A,\left(R_{\lambda}\right)_{\lambda \leq l}$ such that $\forall \lambda \in \llbracket 1, l \rrbracket, R_{\lambda} \subseteq\left(A_{\lambda}^{+} \times A_{\lambda}^{-}\right) \cup\left(A_{\lambda}^{-} \times A_{\lambda}^{+}\right)$, is the graph $(A, R)$ with

$R=\left\{(x, y) \in\left(A^{+} \times A^{-}\right) \cup\left(A^{-} \times A^{+}\right)\right.$and level $(x)<$ level $\left.(y)\right\} \cup \cup_{\lambda \in \llbracket 1, l \rrbracket} R_{\lambda}$

The instantiated universe associated to a bla $A$ given $R$ and given a set of instantiated arguments $I$ included in $I_{A, V, C}$ is a graph $\left(I, R_{I}\right)$ where $R_{I}$ is s.t. $\left(a_{1 v_{1}}\left(c_{1}\right), a_{2 v_{2}}\left(c_{2}\right)\right) \in R_{I}$ iff $c_{1}=c_{2}$ and $a_{1}, a_{2} \in A$ and $\left(a_{1}, a_{2}\right) \in R$.

\footnotetext{
${ }^{2}$ In the literature, the instantiated arguments could be called practical arguments while generic arguments are more related to epistemic arguments.
} 
In other words, an instantiated universe is a graph of instantiated arguments. Each argument is a feature relative to a given candidate according to the opinion of a voter. Attack relations are induced between two arguments that concern the same candidate when the features described in those arguments were consensually said incompatible.

Example 2 This example is inspired from the literature on multi-criteria production planning. The supply chain characteristics and objectives give us concrete features that are used in industrial domain. Here, each argument corresponds to a given industrial objective, then, an argument is enabled for a given production plan if the plan achieves this objective. The definition of arguments is done at the same time as the definition of the bla. We consider that the customer and the supplier deliberate on the basis of the following table A (which is a bla) containing the features that may characterize a plan ${ }^{3}$ :

\begin{tabular}{|c|c|}
\hline$A^{+}$ & $A^{-}$ \\
\hline St: stable load & Rb: risk of back-ordering \\
Ruf: robust under failure & $H i$ : high inventory level \\
Rl: regrouping lot & Me: maintenance expensive \\
$Q f:$ quick prod. flow & $H c i:$ high capacity not used \\
\hline Pd: periodic delivery date & Sc: requires subcontract \\
Qd: constant quantity & $T w:$ necessity of temporary \\
delivered & workers \\
Sp: stable plan & Ot: necessity of overtime \\
& Ic: important impact carbon \\
\hline Pr: satisf. for pers. reason & NPr: non satisfactory \\
Lic: low impact carbon & for personal reason \\
\hline
\end{tabular}

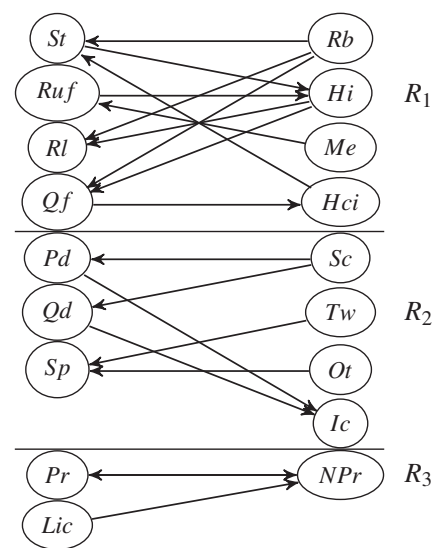

In this bla $A$, the set of arguments is $A=\{S t, R b, R u f, H i, R l, M e, Q f, H c i, P d, S c, Q d, O t, S p, T w, I c$, $\operatorname{Pr}, N P r, L i c\}$. This bla has three levels of importance. The family of attack relations $\left(R_{\lambda}\right)_{\lambda \leq 3}$ between arguments of the same level is s.t. $R_{1}=\{(\mathrm{St}, \mathrm{Hi}),(\mathrm{Rb}, \mathrm{St}),(\mathrm{Rb}, \mathrm{Rl}),(\mathrm{Rb}, \mathrm{Qf}),(\mathrm{Ruf}, \mathrm{Hi}),(\mathrm{Hi}, \mathrm{Rl}),(\mathrm{Hi}, \mathrm{Qf})$, $(\mathrm{Me}, \mathrm{Ruf}),(\mathrm{Qf}, \mathrm{Hci}),(\mathrm{Hci}, \mathrm{St})\}, R_{2}=\{(\mathrm{Pd}, \mathrm{Ic}),(\mathrm{Sc}, \mathrm{Pd}),(\mathrm{Sc}, \mathrm{Qd}),(\mathrm{Qd}, \mathrm{Ic}),(\mathrm{Tw}, \mathrm{Sp}),(\mathrm{Ot}, \mathrm{Sp})\}$ and $R_{3}=\{(\mathrm{Pr}, \mathrm{NPr}),(\mathrm{NPr}, \mathrm{Pr}),(\mathrm{Lic}, \mathrm{NPr})\}$. For sake of clarity, the other attack relations from any argument towards each opposite argument of a greater level are not shown on the picture.

The three leveled bla proposed in this example is consistent with the standard ideas used in the supply chain management domain. This is why we consider at the most important level that the supply chain has to satisfy the final customer (hence avoid the risk of back-ordering Rb). The supply chain should also have a quick flow of production $(Q f)$ (hence the inventory level should not be high: Hi). A good production plan should ensure a proper management of the storage (i.e a stable load St and no waste in the storage capacities Hci). Moreover, the supply chain is supposed to have a low risk of failure (Ruf) and to be cost effective (hence avoid expensive maintenance $(\mathrm{Me})$ and allow for regrouping lots $\mathrm{Rl}$ ). At the second level, it is interesting (but not crucial) to have stability on production and delivery (a stable plan $\mathrm{Sp}$, a constant quantity delivered $Q d$ and periodic delivery dates $P d)$ in order to satisfy the workers unless this could increase the cost or induce some errors. Besides, here, the actors are considering that subcontracting $(\mathrm{Sc})$ increases the risk of back-ordering, hence they prefer not to use overtime (Ot) because it is expensive. Temporary workers $(T w)$ decrease the productivity because they need some learning time. Since the environmental impact has to be integrated in the decision process (according to the idea of "Green SCM"), the actors should consider carbon impact (Ic). The third level takes into account the good impact on environment (Lic) and the actors personal preferences (a plan may be satisfactory - or not-for personal reason $\mathrm{Pr}-\mathrm{NPr}$ ) witch are not mandatory.

Let us justify the attack relation $R_{1}$ of the highest level of this bla, Rb attacks $S t, R l$ and $Q f$ since the primary objective of the supply chain is to fulfill the demand. Rb does not attack Ruf because this feature limits the risk to increase the level of back-ordering. Hi attacks Rl because the actors want to enforce regrouping the lots unless the inventory level becomes to high. The attack between Hi and Qf is different because for a voter the two arguments may coexist (or may be exclusive). So this attack can be described as

\footnotetext{
${ }^{3}$ In real life, the establishment of this table is more complex and depends on the particular objectives and priorities of the suppliers and the customers.
} 
follow "if the plan makes quick flow for one of the voter then it is acceptable only if this plan does not deal with a high inventory level for an other voter". Hi is attacked by St and Ruf because St or Ruf may justify $H$ i. Me attacks Ruf since a robust plan is a benefit only if this robustness is not too expensive to guarantee. $H c i$ is attacked by $Q f$ since it is better to have a quick flow of production than to under-exploit the storage capacity. An example of set of instantiated arguments $I_{B}$ for $c_{1}, c_{2}$ and $c_{3}$ is given below together with a picture of the part of the instantiated bla obtained for candidate $c_{3}$ :

\begin{tabular}{|c|c|c|c|c|c|c|}
\hline & $I^{+}\left(c_{1}\right)$ & $I^{-}\left(c_{1}\right)$ & $I^{+}\left(c_{2}\right)$ & $I^{-}\left(c_{2}\right)$ & $I^{+}\left(c_{3}\right)$ & $I^{-}\left(c_{3}\right)$ \\
\hline 1 & $Q f_{v_{2}}$ & & $\begin{array}{c}R u f_{v_{1}}, Q f_{v_{1}} \\
S t_{v_{1}}\end{array}$ & $\mathrm{Hci}_{v_{2}}$ & & \\
\hline 2 & $S p_{v_{1}}$ & & $P d_{v_{1}}, Q d_{v_{1}}$ & $I c_{v_{2}}$ & $Q d_{v_{2}}, S p_{v_{2}}$ & $T w_{v_{1}}, I c_{v_{1}}$ \\
\hline 3 & $\begin{array}{c}P r_{v_{1}}, P r_{v_{2}}, \\
\quad L i c_{v_{2}} \\
\end{array}$ & & $P r_{v_{1}}, L i c_{v_{1}}$ & $N P r_{v_{2}}$ & $P r_{v_{2}}$ & $N P r_{v_{1}}$ \\
\hline
\end{tabular}

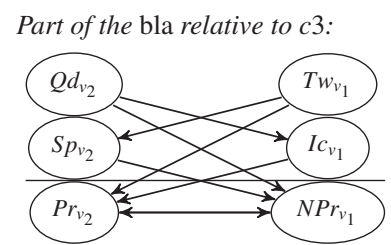

Proposition 1 (compactness of inputs) When the bla contains at least two levels, the framework used to express arguments concerning a set of candidates from the point of view of a set of voters with all the corresponding attacks is more compact ${ }^{4}$ in the bla framework than in a classical argumentation system.

\subsection{Decision protocol}

Given a bla $A$, the set of possible candidates $C$ is given to all voters then each voter $v$ :

- divides privately $C$ into two sets $C_{v}^{+}$and $C_{v}^{-}$,

- instantiates the features concerning each candidate ${ }^{5} c$, collected in $I_{v}(c)$,

- and finally chooses for each candidate the arguments that he wants to present.

For instance, in our example, $v_{1}$ 's private preferences are $C_{v_{1}}^{+}=\left\{c_{1}, c_{2}\right\}, C_{v_{1}}^{-}=\left\{c_{3}\right\}$ and $v 2$ 's ones are $C_{v_{2}}^{+}=$ $\left\{c_{1}, c_{3}\right\}, C_{v_{2}}^{-}=\left\{c_{2}\right\}$. In order to select admissible candidates, we can focus on the existence of non attacked arguments that are in favor and against them. Given a candidate $c$, the set of non-attacked arguments concerning $c$, are denoted $S(c)$ (for "sources"), they are the vertices in $I(c)$ that have no predecessors. We may consider six main cases:

Definition 4 (admissibility status) Given a bla $A$ with an associated instantiated universe $\left(I, R_{I}\right)$, and a candidate $c$, let $S(c)=\{x \in I(c) \mid \forall y,(y, x) \notin R\}$, the status of $c$ is

- necessary admissible $\left(N_{a d}\right)$ if $\varnothing \subset S(c) \subseteq I^{+}(c)$

- possibly admissible $\left(\Pi_{a d}\right)$ if $S(c) \cap I^{+}(c) \neq \varnothing$

- indifferent $\left(I d_{a d}\right)$ if $S(c)=\varnothing$

- controversial $\left(C t_{a d}\right)$ if $S(c) \cap I^{+}(c) \neq \varnothing$ and $S(c) \cap I^{-}(c) \neq \varnothing$

- possibly inadmissible $\left(\Pi_{\neg a d}\right)$ if $S(c) \cap I^{-}(c) \neq \varnothing$

- necessary inadmissible $\left(N_{\neg a d}\right)$ if $\varnothing \subset S(c) \subseteq I^{-}(c)$

In other words, a necessary admissible candidate has an argument in its favor that is unattacked and no unattacked argument against it; a possibly admissible candidate has at least one unattacked argument in its favor. An indifferent candidate has no unattacked arguments in its favor or against it, while a controversial candidate is both supported and criticized by unattacked arguments. A candidate is possibly inadmissible if there is an unattacked argument against it and necessary inadmissible if there is an unattacked argument against it and no unattacked argument in favor of it.

Example 3 The arguments given by $v_{1}$ and $v_{2}$ about $c_{1}$ belong only to $I^{+}$, thus $c_{1} \in N_{\text {ad }}$, they seem to agree to select candidate $c_{1} . c_{2}$ is also in $N_{a d}, c_{3}$ is only possibly admissible $\left(c_{3} \in \Pi_{a d}\right)$ because $Q d_{v_{2}}$ and $T w_{v_{1}}$ are not attacked.

The above definition is related to possibility theory $[18,6]$, where necessary (resp. possibly) admissible could be understood as it is certain (resp. possible) that the candidate is admissible. The indifference

\footnotetext{
${ }^{4}$ in terms of number of symbols used to represent the same information in the worst case

${ }^{5}$ In this paper we suppose that a voter $v$ uses the following basic strategy: if $c \in C_{v}^{+}$then he only gives arguments in $I^{+}(c)$, if $c \in C_{v}^{-}$then he only gives arguments in $I^{-}(c)$.
} 
case is linked to an impossibility to have unattacked arguments in favor and against a candidate, thus an impossibility to decide. However it is not related to a standard definition of possibilistic ignorance about the admissibility of a candidate, which rather corresponds to a controversial candidate that is both possibly admissible and possibly inadmissible. With these definitions we get:

Proposition 2 Inclusion and Duality:

- $N_{a d} \subseteq \Pi_{a d}$ and $N_{\neg a d} \subseteq \Pi_{\neg a d}$

- $C t_{a d}=\Pi_{a d} \cap \Pi_{\neg a d}$ and $I d_{a d}=\left(C \backslash \Pi_{a d}\right) \cap\left(C \backslash \Pi_{\neg a d}\right)$

- $N_{a d}=C \backslash\left(\Pi_{\neg a d} \cup I d_{a d}\right)$ and $N_{\neg a d}=C \backslash\left(\Pi_{a d} \cup I d_{a d}\right)$.

The following property reveals that if a candidate is acceptable for all the voters then it is necessary admissible, similarly, if a candidate is unacceptable for all the voters then it is necessary inadmissible.

Proposition 3 (unanimity) Considering a set of voters $V$ using the basic strategy, for any candidate $c \in C$

- If $\forall v \in V, c \in C^{+}(v)$ and $I^{+}(c) \neq \varnothing$ then $c \in N_{a d}$.

- If $\forall v \in V, c \in C^{-}(v)$ and $I^{-}(c) \neq \varnothing$ then $c \in N_{\neg a d}$.

With these admissibility status, we can propose 3 thresholds of admissibility (from the strongest to the weakest): the first threshold contains the candidates such that all the unattacked arguments about them are positive, the second threshold can be divided into two sets: $2 \mathrm{a}$ is the set of candidates under the first threshold together with the candidates for which no unattacked argument concerning them is available (neither positive nor negative), the set $2 \mathrm{~b}$ tolerates candidates that are concerned by negative unattacked argument provided that they are also concerned at least by one positive unattacked argument. The third threshold is the union of the sets $1,2 \mathrm{a}$ and $2 \mathrm{~b}$ as shown in the figure below:

- threshold 1: $c \in N_{a d}$

- threshold 2a: $c \in N_{a d} \cup I d_{a d}$ (or in $C \backslash \Pi_{\neg a d}$ )

- threshold 2b: $c \in \Pi_{a d}$ (or in $N_{a d} \cup C t_{a d}$ )

- threshold 3: $c \in \Pi_{a d} \cup I d_{a d}$ (or in $C \backslash N_{\neg a d}$ )

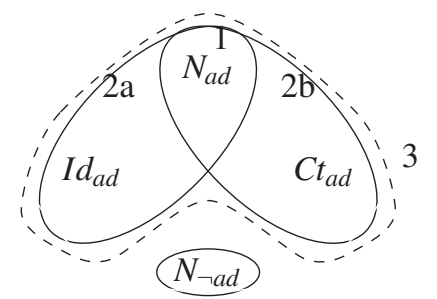

Note that qualitative explanations of the selection can be drawn on a graph, they are simple and clear compared to quantitative explanations (that may result from complex and obscure numeric computation and that are not necessarily based on a consensual agreement).

How to compute the admissible sets? Given a candidate $c$, we first consider the set of unattacked arguments concerning $c$ (i.e. $S(c)=\{x \in I(c) \mid \forall y,(y, x) \notin R$ ) and then according to this set, we may assign one of the six status (necessary/possibly admissible/inadmissible, indifferent/controversial) to $c$ by using Def. 4 . Building the sets $N_{a d}, \Pi_{a d}, I d_{a d}, N_{\neg a d}$ and $\Pi_{\neg a d}$ can be done in polynomial time wrt $|I|$.

\section{Conclusion}

This paper introduces a new approach for SCM collaborative planning which is the basis of a new framework for collaborative group decision making. The proposed process is a kind of bipolar argumentative vote with more expressive power than standard vote procedures but also a more compact representation of an argumentation system which focus on admissibility of candidate and not on a ranking.

There are numerous benefits of this new decision process. First, our framework follows the standard principles defined by $\mathrm{CPFR} R$ that are commonly used by industrialists. Moreover our proposal handles multi-criteria expression which is often not the case for classical approaches or done roughly by using arbitrary aggregation functions. Furthermore, it guarantees a succinct expression. And finally, the qualitative aspect of this approach enables to justify the decision in an understandable and clear way to the actors. However there are some drawbacks, for instance the need to define a protocol for reaching a bla and a common definition of conflicts and priorities which maybe a difficult task (but it has only to be done once). This difficulty may be increased in domains with more open criteria than in the SCM field where standard 
criteria are already well established. In order to complete the definition of this bipolar deliberation protocol, a study is still needed to establish that the admissible sets are rational regarding classical decision rules such as Pareto, BiPoss and BiLexi [4].

\section{References}

[1] M. Albrecht. Supply Chain Coordination Mechanisms: New Approaches for Collaborative Planning. Lecture Notes in Eco. and Math. Systems, 628. Springer Berlin Heidelberg, 2010.

[2] L. Amgoud and S. Vesic. A formal analysis of the role of argumentation in negotiation dialogues. Journal of Logic and Computation, 22:957-978, octobre 2012.

[3] A. Baykasoglu and T. Gocken. Multi-objective aggregate production planning with fuzzy parameters. Advances in Engineering Software, 41(9):1124-1131, 2010.

[4] JF. Bonnefon, D. Dubois, and H. Fargier. An overview of bipolar qualitative decision rules. In G. Della Riccia, D. Dubois, R. Kruse, and H-J. Lenz, editors, Preferences and Similarities, volume 504 of CISM Courses and Lectures, pages 47-73. Springer, 2008.

[5] M. Christopher. Logistics And Supply Chain Management: Creating Value-Adding Networks. Pearson Education, 2005.

[6] D. Dubois and H. Prade. Possibility theory: qualitative and quantitative aspects. In Quantified Representation of Uncertainty and Imprecision, volume 1 of Handbook of Defeasible Reasoning and Uncertainty Management Systems, pages 169-226. Kluwer Academic, 1998.

[7] G. Dudek. Collaborative Planning in Supply Chains: A Negotiation-based Approach. Springer-Verlag Berlin Heidelberg, 2009.

[8] P. M. Dung. On the acceptability of arguments and its fundamental role in nonmonotonic reasoning, logic programming and n-person games. Artificial Intelligence, 77:321-357, 1995.

[9] R. Guillaume, B. Grabot, and C. Thierry. Management of the risk of backorders in a mto-ato/mts context under imperfect requirements. Applied Mathematical Modelling, 2013.

[10] R.K. Ireland and C. Crum. Supply Chain Collaboration: How To Implement CPFR And Other Best Collaborative Practices. Integrated Business Management Series. J. Ross Publishing, Incorporated, 2005.

[11] A. Jamalnia and M. Soukhakian. A hybrid fuzzy goal programming approach with different goal priorities to aggregate production planning. Comp. Ind. Eng., 56(4):1474-1486, 2009.

[12] Ta-Ping Lu, Amy J.C. Trappey, Yi-Kuang Chen, and Yu-Da Chang. Collaborative design and analysis of supply chain network management key processes model. Journal of Network and Computer Applications, 2013.

[13] F. Marcotte, B. Grabot, and R. Affonso. Cooperation models for supply chain management. International Journal of Logistics Systems and Management, 5(1):123-153, January 2009.

[14] I. Rahwan, S. D. Ramchurn, N. R. Jennings, P. McBurney, S. Parsons, and L. Sonenberg. Argumentation-based negotiation. Knowledge engineering review, 18(4):343-375, 2003.

[15] H. Selim, C. Araz, and I. Ozkarahan. Collaborative production-distribution planning in supply chain: A fuzzy goal programming approach. Transportation Research Part E: Logistics and Transportation Review, 44(3):396 419, 2008.

[16] P. Slovic, M. Finucane, E. Peters, and D. MacGregor. Rational actors or rational fools? Implications of the affect heuristic for behavioral economics. The Journal of Socio-Economics, 31:329-342, 2002.

[17] D. N. Walton and E. C. W. Krabbe. Commitment in Dialogue: Basic Concepts of Interpersonal Reasoning. State University of New York Press, Albany, NY, 1995.

[18] L.A. Zadeh. Fuzzy Sets as a Basis for a Theory of Possibility. Memorandum: Electronics Research Laboratory. College of Eng., University of California, 1977. 